\title{
Competition Jumping Horses: Effects of Age, Sex and Breed on the Fei/Wbfsh World Ranking
}

\author{
Anca ROMAN-POPOVICI*, Dan SUMOVSCHI, Ioan GÎLCĂ \\ Faculty of Animal Science, University of Agricultural Science and Veterinary Medicine \\ "Ion Ionescu de la Brad", 3 Mihail Sadoveanu Alley, 700490 Iaşi, Romania \\ *Corresponding author, email: roman_anca2004@yahoo.com
}

Bulletin UASVM Animal Science and Biotechnologies 72(1) / 2015

Print ISSN 1843-5262; Electronic ISSN 1843-536X

DOI:10.15835/buasvmcn-asb:10500

\begin{abstract}
The breed, age and sex of the horses are all important factors in determining the future rank in international high-level show jumping competition and this is the reason why we choose to analyze these factors. The objective of this study was to analyze the differences between the top jumping horses in the world regarding breed, sex and age, and to investigate the impact of these factors on the average number of FEI points gained in the last year. For this research, we examine the first 103 jumping horses from FEI\&WBFSH World Ranking List for Jumping Horses April 2014 , divided in 6 groups for breed variable and 3 groups for sex and age variables. The results show that $82.5 \%$ of the horses have between 10 and 14 years, 10.7\% have less than 10 years and only $6.8 \%$ have more than 14 years. For breed variable, the results show that $34 \%$ of the horses belong to warmblood breeds from Germany $(34.3 \%$ HOLST, 20\% OLDBG, 20\% WESTF, 17.1\% HANN, 5.7\% BRAND and 2.9\% Thuringer), 26.2\% belong to warmblood breeds from Belgium (11.1\% ZANG, 70.4\% BWP and 18.5\% SBS), 18.4\% belong to warmblood breeds from The Nederland (94.7\% KWPN and 5.3\% NRPS), 9.7\% belong to warmblood breeds from Great Britain (70\% AES, 20\% ISH and 10\% SSH), $8.7 \%$ belong to warmblood breeds from France (100\% SF) and 2.9\% belong to warmblood breeds from Sweden (100\% SWB). The average number of FEI points gain in the last year was evaluated as not being significantly different ( $p>0.05$ ) for any of the variable considered for this study. In the present research work, we demonstrated that for performance in show jumping competition at the highest levels it is necessary a horse from a sport breed with a strict selection and not one from a traditional breed, and that between the principal warmblood breeds grown in Europe there are no significant differences.
\end{abstract}

Keywords: breed, jumping, ranking, sport horses, warmblood

\section{INTRODUCTION}

In the latest years, interest in sport horses and in horse breeding increased. Many new breeds arise from the general demand for good animals with a good attitude and performance in sports and the market of sport horses for show jumping competition become more and more international (Ruhlmann et al., 2009). Obtaining performance at the highest level in show jumping depends not only on the abilities of the trainer and rider, but mostly of the horse. For this reason, a sport horse should ideally display certain qualities of temperament and physiognomy. Sport horses typically share many physical features, including sloping shoulders, an uphill build, and an arched neck (Schacht, 2012).

The purpose and breeding of sport horses across the world varies little and in this way, the warmbloods breedstend to take over the equestrian world especially in traditional equestrian sporting events, such as show jumping, eventing, and dressage (Bartolomé et al., 2011). Warmbloods are a group of middleweight horse types and breeds, primarily originating in Europe, registered with organizations that are characterized by open studbook policy, studbook selection, and the aim 
of breeding for equestrian sport. The warmblood breeds are named according to the individual country from which they are originated.

Taking into account the above, we can say that the breed, age and sex of the horses are all important factors in determining the future rank in international high-level show jumping competition and this is the reason why we choose to analyze these factors.

\section{MATERIALS AND METHODS}

Animals. For this research we examine the first 103 show jumping horses from FEI\&WBFSH World Ranking List for Jumping Horses April 2014 (http://www.wbfsh.org), divided in 6 groups for breed variable and 3 groups for sex and age variables. The three categories for the sex variable were: stallions (s), geldings (g) and mares (m), while for the age variable there are: horses under 10 years ( $<10$ years), horses aged between 10 to
14 years $(10-14$ years $)$ and horses older than 14 years ( $>14$ years), the divisions being dictated by the fact that horse jumping peak of form is obtained within the range of age 10 to 14 years.

The breed variable was divided into six categories:

- warmblood breeds from Germany including: Holsteiner (HOLST), Oldenburg (OLDBG), Westphalian (WESTF), Hanoverian (HANN), Brandenburger (BRAND) and Thüringer Reitpferd (Thuringer);

- warmblood breeds from Belgium including: Zangersheide (ZANG), Belgian Warmblood (BWP) and Belgian Sport Horse (SBS);

- warmblood breeds from The Nederland including: Dutch Warmblood (KWPN) and Dutch Riding Horse (NRPS);

- warmblood breeds from Great Britain including: Anglo-European (AES), Irish Sport Horses (ISH) and Scottish Sports Horse (SSH);

Tab. 1. Horse distribution according to gender, age and breed variables

\begin{tabular}{|c|c|c|c|c|c|c|c|c|}
\hline & \multirow{2}{*}{ Breed } & \multicolumn{3}{|c|}{ Age } & \multicolumn{3}{|c|}{ Sex } & \multirow{2}{*}{$\mathrm{N}$} \\
\hline & & $<10$ years & $10-14$ years & $>14$ years & $\mathrm{m}$ & $\mathrm{s}$ & $\mathrm{g}$ & \\
\hline & HOLST & 1 & 10 & 1 & 3 & 6 & 3 & 12 \\
\hline & OLDBG & 1 & 6 & 0 & 2 & 0 & 5 & 7 \\
\hline & WESTF & 0 & 6 & 1 & 0 & 2 & 5 & 7 \\
\hline \multirow{4}{*}{ WBG } & HANN & 0 & 6 & 0 & 2 & 3 & 1 & 6 \\
\hline & BRAND & 0 & 2 & 0 & 0 & 0 & 2 & 2 \\
\hline & Thuringer & 0 & 1 & 0 & 1 & 0 & 0 & 1 \\
\hline & Total & 2 & 31 & 2 & 8 & 11 & 16 & 35 \\
\hline \multirow{4}{*}{ WBB } & ZANG & 0 & 3 & 0 & 2 & 0 & 1 & 3 \\
\hline & BWP & 5 & 13 & 1 & 8 & 1 & 10 & 19 \\
\hline & SBS & 0 & 5 & 0 & 0 & 2 & 3 & 5 \\
\hline & Total & 5 & 21 & 1 & 10 & 3 & 14 & 27 \\
\hline \multirow{3}{*}{ WBN } & KWPN & 3 & 12 & 3 & 4 & 2 & 12 & 18 \\
\hline & NRPS & 0 & 1 & 0 & 0 & 1 & 0 & 1 \\
\hline & Total & 3 & 13 & 3 & 4 & 3 & 12 & 19 \\
\hline \multirow{4}{*}{ WBGB } & AES & 0 & 6 & 1 & 5 & 1 & 1 & 7 \\
\hline & ISH & 0 & 2 & 0 & 1 & 0 & 1 & 2 \\
\hline & SSH & 0 & 1 & 0 & 1 & 0 & 0 & 1 \\
\hline & Total & 0 & 9 & 1 & 7 & 1 & 2 & 10 \\
\hline \multirow{2}{*}{ WBF } & SF & 1 & 8 & 0 & 3 & 4 & 2 & 9 \\
\hline & Total & 1 & 8 & 0 & 3 & 4 & 2 & 9 \\
\hline \multirow{2}{*}{ WBS } & SWB & 0 & 3 & 0 & 2 & 1 & 0 & 3 \\
\hline & Total & 0 & 3 & 0 & 2 & 1 & 0 & 3 \\
\hline
\end{tabular}

Note: WBG: warmblood breeds from Germany, WBN: warmblood breeds from The Netherlands, WBF: warmblood breeds from France, WBB: warmblood breeds from Belgium, WBGB: warmblood breeds from Great Britain, WBS: warmblood breeds from Sweden, N: total number. 
- warmblood breeds from France including: Selle Français (SF);

- warmblood breeds from Sweden including: Swedish warmblood (SWB).

The horses' distribution for the three variables taken into account (sex, age and breed) is shown in Table 1.

Statistical analysis. Data was manipulated using Excel 2007 (Microsoft) and was analyzed using SPSS Version 21 for Windows (IBM, USA). The aim of the statistical analysis was to analyze the differences between the top jumping horses in the world regarding breed, sex and age, and to investigate the impact of these factors on the average number of FEI points gained in the last year. The statistical test used was:

- One-Way ANOVA for comparing the means (using the F distribution) of our three to six categories for each variable;

- Two-Way Factorial ANOVA for studying the effect of two independent categorical variables on the dependent variable. We test the main effect of each independent variable and also test if the effect of one independent variable on the dependent variable is the same across all level of the other independent variable, that is, if there is any interaction between the independent variables.

The value of alpha was set at 0.05 for all statistical tests.

\section{RESULTS AND DISCUSSION}

The results show that the number of FEI points gained in the last year for the first 103 jumping horses from FEI\&WBFSH World Ranking List for Jumping Horses April 2014 vary between 365 and 1104 , with an average of 516.82 .

Age. The results show that $82.5 \%$ of the horses have between 10 and 14 years, $10.7 \%$ have less than 10 years and only $6.8 \%$ have more than 14 years. For the age variable, the results from the statistical analysis show that the average number of FEI points gained in the last year doesn't present statistically significant differences for the 0.05 significance level $(F=2.935, p=0.058)$. The descriptive statistics and One-way ANOVA main statistics for the three dimensions of age variable regarding the average number of FEI points gained in the last year are shown in Table 2 and 3.

Therefore, we can say that, regarding the average number of FEI points gained in the last year, there are no statistically significant differences between horses aged over 14, horses aged between 10 and 14 years and horses younger than 10 years. The results can be explained by the fact that the majority of horses (82.5\%) were in the group age between 10 and 14 years and because the horses younger than 10 years were all 9 years old so the variability between this two groups is really small.

Tab. 2. The descriptive statistics for age variable regarding the average number of FEI points gained in the last year

\begin{tabular}{|c|c|c|c|c|c|c|c|c|}
\hline & $\mathrm{N}$ & Mean & $\begin{array}{l}\text { Std. } \\
\text { Deviation }\end{array}$ & Std. Error & $\begin{array}{l}\text { 95\% Con } \\
\text { for Mean } \\
\text { Lower } \\
\text { Bound }\end{array}$ & $\begin{array}{l}\text { ence Inte } \\
\text { Upper } \\
\text { Bound }\end{array}$ & -Minimum & Maximum \\
\hline over 14 years & 7 & 587.57 & 130.331 & 49.261 & 467.03 & 708.11 & 460 & 840 \\
\hline $\begin{array}{l}\text { between } 10 \text { anc } \\
14 \text { years }\end{array}$ & 85 & 523.49 & 165.189 & 17.917 & 487.86 & 559.12 & 365 & 1104 \\
\hline under 10 years & 11 & 420.18 & 41.417 & 12.488 & 392.36 & 448.01 & 365 & 473 \\
\hline Total & 103 & 516.82 & 158.199 & 15.588 & 485.90 & 547.73 & 365 & 1104 \\
\hline
\end{tabular}

Tab. 3. One-way ANOVA main statistics for the three dimensions of age variable regarding the average number of FEI points gained in the last year

\begin{tabular}{cccccc}
\hline & Sum of Squares & df & Mean Square & F & Sig. \\
\hline Between Groups & 141554.897 & 2 & 70777.449 & 2.935 & 0.058 \\
Within Groups & 2411206.598 & 100 & 24112.066 & & \\
\hline Total & 2552761.495 & 102 & & & \\
\hline
\end{tabular}


Tab. 4. The descriptive statistics for sex variable regarding the average number of FEI points gained in the last year

\begin{tabular}{|c|c|c|c|c|c|c|c|c|}
\hline & \multirow{2}{*}{$\mathrm{N}$} & \multirow{2}{*}{ Mean } & \multirow{2}{*}{ Std. Deviation } & \multicolumn{3}{|c|}{$95 \%$ Confidence Interval for Mean } & \multirow{2}{*}{ Minimum } & \multirow{2}{*}{ Maximum } \\
\hline & & & & תน. L1101 & Lower Bound & Upper Bound & & \\
\hline mare & 34 & 526.76 & 183.549 & 31.478 & 462.72 & 590.81 & 365 & 1104 \\
\hline stallion & 23 & 541.35 & 176.698 & 36.844 & 464.94 & 617.76 & 365 & 954 \\
\hline gelding & 46 & 497.20 & 126.419 & 18.639 & 459.65 & 534.74 & 365 & 879 \\
\hline Total & 103 & 516.82 & 158.199 & 15.588 & 485.90 & 547.73 & 365 & 1104 \\
\hline
\end{tabular}

Tab. 5. One-way ANOVA main statistics for the three dimensions of sex variable regarding the average number of FEI points gained in the last year

\begin{tabular}{cccccc}
\hline & Sum of Squares & df & Mean Square & F & Sig. \\
\hline Between Groups & 34914.921 & 2 & 17457.460 & 0.693 & 0.502 \\
Within Groups & 2517846.574 & 100 & 25178.466 & & \\
\hline Total & 2552761.495 & 102 & & & \\
\hline
\end{tabular}

Tab. 6. The descriptive statistics for breed variable regarding the average number of FEI points gained in the last year

\begin{tabular}{|c|c|c|c|c|c|c|c|c|}
\hline & \multirow{2}{*}{$\mathrm{N}$} & \multirow{2}{*}{ Mean } & \multirow{2}{*}{\multicolumn{2}{|c|}{ Std. Deviation Std. Error }} & \multicolumn{2}{|c|}{ 95\% Confidence Interval for Mean } & \multirow{2}{*}{ Minimum } & \multirow{2}{*}{ Maximum } \\
\hline & & & & & Lower Bound & Upper Bound & & \\
\hline WBG & 35 & 522.66 & 167.598 & 28.329 & 465.09 & 580.23 & 365 & 954 \\
\hline WBN & 19 & 499.79 & 122.305 & 28.059 & 440.84 & 558.74 & 365 & 840 \\
\hline WBB & 27 & 491.37 & 132.418 & 25.484 & 438.99 & 543.75 & 365 & 830 \\
\hline WBF & 9 & 524.00 & 142.927 & 47.642 & 414.14 & 633.86 & 405 & 863 \\
\hline WBS & 3 & 645.00 & 190.591 & 110.038 & 171.55 & 1118.45 & 425 & 760 \\
\hline WBGB & 10 & 552.50 & 247.168 & 78.161 & 375.69 & 729.31 & 365 & 1104 \\
\hline Total & 103 & 516.82 & 158.199 & 15.588 & 485.90 & 547.73 & 365 & 1104 \\
\hline
\end{tabular}

Tab. 7. One-way ANOVA main statistics for the six dimensions of breed variable regarding the average number of FEI points gained in the last year

\begin{tabular}{cccccc}
\hline & Sum of Squares & df & Mean Square & F & Sig. \\
\hline Between Groups & 86675.655 & 5 & 17335.131 & 0.682 & 0.638 \\
Within Groups & 2466085.840 & 97 & 25423.565 & & \\
\hline Total & 2552761.495 & 102 & & & \\
\hline
\end{tabular}


Sex. The results show that $33 \%$ of the horses are mares, $22.3 \%$ are stallions and $44.7 \%$ are geldings. For the sex variable the results from the statistical analysis shows that the average number of FEI points gained in the last year doesn't present statistically significant differences for the 0.05 significance level $(\mathrm{F}=0.693, \mathrm{p}=0.502)$. The descriptive statistics and One-way ANOVA main statistics for the three dimensions of sex variable regarding the average number of FEI points gained in the last year are shown in Table 4 and 5. Therefore, we can say that, regarding the average number of FEI points gained in the last year there are no statistically significant differences between mares, stallions and geldings.

Breed. The results show that 34\% of the horses belong to warmblood breeds from Germany (34.3\% HOLST, 20\% OLDBG, 20\% WESTF, 17.1\% HANN, 5.7\% BRAND and 2.9\% Thuringer), 26.2\% belong to warmblood breeds from Belgium (11.1\% ZANG, 70.4\% BWP and 18.5\% SBS), $18.4 \%$ belong to warmblood breeds from The Nederland (94.7\% KWPN and 5.3\% NRPS), 9.7\% belong to warmblood breeds from Great Britain (70\% AES, 20\% ISH and 10\% SSH), 8.7\% belong to warmblood breeds from France $(100 \%$ SF) and $2.9 \%$ belong to warmblood breeds from Sweden (100\% SWB).

For the breed variable, the results from the statistical analysis show that the average number of FEI points gained in the last year doesn't present statistically significant differences for the 0.05 significance level $(\mathrm{F}=0.682, \mathrm{p}=0.638)$. The descriptive statistics and One-way ANOVA main statistics for the three dimensions of breed variable regarding the average number of FEI points gained in the last year are shown in Table 6 and 7. Therefore, we can say that, regarding the average number of FEI points gained in the last year, there are no statistically significant differences between the six warmblood horse breeds considered in this study. These results are not surprising at all, because in case of all warmblood sport horses from this list are used breeding schemes that share the same objectives even if the procedure for selection varies in different European countries. Another explanation is that all warmblood breeds have open study books and mandy stallion are used as breeding stallions in more that one breed (Dubois, 2008).

Sex $X$ Age. The results from the statistical analysis (Two-Way Factorial ANOVA) shows that there are no main effects for none of the two variable taken to be studied (sex and age) and doesn't exist a statistically significant interaction effect between sex and age variables regarding the average number of FEI points gained in the last year. The Two-way factorial ANOVA main statistics for sex and age variables regarding the average number of FEI points gained in the last year are shown in Table 8.

Tab. 8. Two-Way Factorial ANOVA Tests of Between-Subjects Effects. Effects of sex and age variables on the average number of FEI points gained in the last year

\begin{tabular}{cccccc}
\hline Source & $\begin{array}{c}\text { Type III Sum of } \\
\text { Squares }\end{array}$ & df & Mean Square & F & Sig. \\
\hline Corrected Model & $166026.923^{a}$ & 7 & 23718.132 & .944 & .477 \\
Intercept & 7350537.733 & 1 & 7350537.733 & 292.576 & .000 \\
sex & 16599.240 & 2 & 8299.620 & .330 & .719 \\
age & 47710.288 & 2 & 23855.144 & .950 & .391 \\
sex * age & 18828.802 & 3 & 6276.267 & .250 & .861 \\
Error & 2386734.572 & 95 & 25123.522 & & \\
\hline Total & 30063886.000 & 103 & & & \\
\hline Corrected Total & 2552761.495 & 102 & & & \\
\hline a. R Squared $=.065$ (Adjusted R Squared $=-.004)$ &
\end{tabular}


Breed $\boldsymbol{X}$ Age. The results from the statistical analysis (Two-Way Factorial ANOVA) shows that there are no main effects for none of the two variable taken to be studied (breed and age) and doesn't exist a statistically significant interaction effect between breed and age variables regarding the average number of FEI points gained in the last year. The Two-way factorial ANOVA main statistics for breed and age variables regarding the average number of FEI points gained in the last year is shown in Table 9.
Breed $\boldsymbol{X}$ Sex. The results from the statistical analysis (Two-Way Factorial ANOVA) shows that there are no main effects for none of the two variable taken to be studied (breed and sex) and doesn't exist a statistically significant interaction effect between breed and sex variables regarding the average number of FEI points gained in the last year. The Two-way factorial ANOVA main statistics for breed and sex variables regarding the average number of FEI points gained in the last year is shown in Table 10.

Tab. 9. Two-Way Factorial ANOVA Tests of Between-Subjects Effects. Effects of breed and age variables on the average number of FEI points gained in the last year

\begin{tabular}{cccccc}
\hline Source & $\begin{array}{c}\text { Type III Sum of } \\
\text { Squares }\end{array}$ & df & Mean Square & F & Sig. \\
\hline Corrected Model & $280373.950^{\mathrm{a}}$ & 13 & 21567.227 & .845 & .613 \\
Intercept & 6534230.718 & 1 & 6534230.718 & 255.919 & .000 \\
breed & 56592.028 & 5 & 11318.406 & .443 & .817 \\
age & 90778.259 & 2 & 45389.130 & 1.778 & .175 \\
breed ${ }^{*}$ age & 74025.812 & 6 & 12337.635 & .483 & .819 \\
Error & 2272387.545 & 89 & 25532.444 & & \\
\hline Total & 30063886.000 & 103 & & & \\
\hline Corrected Total & 2552761.495 & 102 & & & \\
\hline a. R Squared $=.110$ (Adjusted R Squared $=-.020)$ &
\end{tabular}

Tab. 10. Two-Way Factorial ANOVA Tests of Between-Subjects Effects. Effects of breed and sex variables on the average number of FEI points gained in the last year

\begin{tabular}{cccccc}
\hline Source & $\begin{array}{c}\text { Type III Sum of } \\
\text { Squares }\end{array}$ & df & Mean Square & F & Sig. \\
\hline Corrected Model & $326056.171^{\mathrm{a}}$ & 16 & 20378.511 & .787 & .696 \\
Intercept & 12992218.287 & 1 & 12992218.287 & 501.787 & .000 \\
breed & 41642.260 & 5 & 8328.452 & .322 & .899 \\
sex & 47065.864 & 2 & 23532.932 & .909 & .407 \\
breed ${ }^{*}$ sex & 223783.593 & 9 & 24864.844 & .960 & .478 \\
Error & 2226705.324 & 86 & 25891.922 & & \\
\hline Total & 30063886.000 & 103 & & & \\
\hline Corrected Total & 2552761.495 & 102 & & & \\
\hline a. R Squared $=.128$ (Adjusted R Squared $=-.035)$ &
\end{tabular}




\section{CONCLUSION}

In conclusion, we can say that regarding the average number of FEI points gained in the last year for neither one of the three variables taken into account (sex, age and breed) there were no statistically significant differences between the first 103 jumping horses from FEI\&WBFSH World Ranking List for Jumping Horses April 2014 considered in this study.

These results are not surprising at all because when we consider the first 103 jumping horses in the world it is normal to exist a great degree of homogeneity especially regarding the breed and age.

For success in the modern show jumping world is recommended to have a horse that belong to a warmblood breed with a strict selection and not a horse from other traditional breeds. Even if breeds like the Thoroughbred had known success in the show jumping arena in the past, the present results show that horses from this breed are no longer present in the first 100 in the world, but for the modern sport horse breeds of the Thoroughbred influence will persist in the future.
Acknowledgments. This paper was published under the frame of European Social Fund, Human Resources Development Operational Programme 2007-2013, project no. POSDRU/ 159/1.5/S/132765.

\section{REFERENCES}

1. Bartolomé E, Cervantes I, Valera M, Gutiérrez JP (2011). Influence of foreign breeds on the genetic structure of the Spanish Sport Horse population. Livestock Science 142: 70-79.

2. Dubois C, Manfredi E, Ricard A (2008). Optimization of breeding schemes for sport horses. Livestock Science 118: 99-112.

3. Ruhlmann C, Janssens S, Philipsson J, Thorén-Hellsten E, Crolly H, Quinn K, Manfredi E, Ricard A (2009). Genetic correlations between horses show jumping competition traits in five European countries. Livestock Science 122: 234-240.

4. Schacht Christian (2012). Sport Horse Conformation: Evaluating Athletic Potential in Dressage, Jumping and Event Prospects. Trafalgar Square Books, Vermont.

5. http://www.wbfsh.org 